\title{
Factors contributing to non-adherence to insulin therapy among type 1 and type2 diabetes mellitus patients in Asser region, Saudi Arabia.
}

\author{
Dalia Almaghaslah*, Arwa Khled Abdelrhman, Shroouk Khaled AL-Masdaf, Layla Mohammed \\ Majrashi, Basayer Mostafa Matary, Wegdan Mohammed Asiri, Bayan Ali Alqhatani
}

King Khalid University, College of Pharmacy, PO Box 1882, Abha, Saudi Arabia

\begin{abstract}
Objective: The aim of this study was to assess the treatment adherence for DM as related to quality of life and to assess the factors contributing to non-adherence among the types 1 and 2 diabetes patients in the Asser Region, Saudi Arabia.

Patients and methods: A cross-sectional study was carried out on 200 DM patients. An eight-item Morisky medication adherence questionnaire was used to assess adherence. A validated questionnaire was used to collect information on factors influencing non- adherence. The percentage of adherence and the factors contributing to non-adherence were analysed with descriptive statistics, chi-square test and independent sample t-test.

Results: The current study showed that the overall prevalence of therapeutic non- adherence was 76 patients $(38 \%)$. Among the non-adherent patients, $(28.9 \%)$ patients were illiterate, 40 had economic problems which prohibited their purchase of the prescribed medicines $(\mathrm{p}=\mathbf{0 . 5 6}),(26 \%)$ patients were aware of the outcomes if the medications were not taken regularly $(\mathrm{p}=0.591)$, most of the patients $(56$, $28.6 \%)$ were not aware of the common side effects of their medications $(p=0.606),(23.5 \%)$ of the patients were not exercising regularly $(p=0.651),(20 \%)$ patients of the low adherent patients were afraid of hypoglycaemia and $(\mathbf{4 2 . 9 \%})$ patients were suffering from forgetfulness in regard to taking their medication.

Conclusion: The current study suggests acceptable level of adherence among diabetic patients in Aseer region, Saudi Arabia. The study finding that the most common barriers to patients' adherence were forgetfulness, fear of hypoglycaemia, weight gain and difficulties with injections.
\end{abstract}

Keywords: Factors to non-adherence, Type I diabetes, Type II diabetes, Saudi Arabia.

Accepted on April 3, 2018

\section{Introduction}

Diabetes mellitus is one of the fastest growing health problems in the world and it is a challenging disease to be managed successfully. Many patients do not achieve good control of their disease, as they do not follow the instructions provided by the health care providers. Avoiding diabetes-related complications could only be achieved if patients adhered to their treatment recommendations [1].

In 2015, the global prevalence of diabetes mellitus was estimated to be 415 million people; by 2040 the prevalence is expected to rise to 642 million [2].

The prevalence of DM in Saudi Arabia was three times higher than the rest of the world [3]. There were 3,852.000 cases of diabetes in Saudi Arabia in 2017. At the moment, 1 in ten adults has diabetes and this expected to continue until 2040 $[2,3]$.
Diabetes and its complications are major causes of death in most countries. In Saudi Arabia, 5 million people died from diabetes in 2017 [2].

Globally, the health expenditure on diabetic patients is generally two -three times higher than the non-diabetic people. It was found that $12 \%$ of global health expenditure was on diabetes $[4,5]$.

The increasing number of diabetes patients is costly and creates a large economic burden on individuals, families, and national health systems. Therefore, attention should be paid to patients with diabetes in order to improve their metabolic control and delay their micro vascular complications [3].

Adherence to the medical regimen continues to rank as a major clinical problem in the management of patients with Diabetes, as in other conditions treated with drugs and life-style modification [5]. Adherence was defined as "active, collaborative and voluntary involvement of the patients in a mutually acceptable course of behaviour to produce a therapeutic result." [6]. 
The Barriers to medication adherence can be classified into two categories; patient related factors such as: difficulty remembering to take their medications, fear of taking medications, depression, and difficulty obtaining refills from the clinician or the pharmacy, or their health beliefs about medications. Other, barriers may be related to the medication itself, such as the complexity of the medication regimen, the drugs administrated in multiple daily dosing, the cost, and the side effects [1].

Several studies around the world showed that the prevalence of poor adherence in diabetes could be as high as $77 \%$. Factors contributed to poor adherence were therapy-related factors as well as patients' related factors [6-8].

Studies evaluating diabetic patient's adherence to treatment in Saudi Arabia are limited, and almost all the studies were focusing on measuring the prevalence of the disease in the country compared to the world. The aim of this study is to assess the factors the contributed to non-adherence in diabetes in Aseer region, Saudi Arabia [9].

\section{Methods}

\section{Patients and study design}

The present cross-sectional study was conducted after obtaining institutional ethics committee approval. The study included 200 patients attending the diabetic centres at Asser Hospital, Saudi Arabia between February 2017 and May 2017.

\section{Inclusion criteria}

Adults with types 1 or 2 diabetes of genders, being treated with insulin and/or orally administrated drugs (monotherapy and/or in association with other therapies). Additionally, patients who were able to maintain dialogues were included.

\section{Exclusion criteria}

People less than 18 y old, pregnant women with gestational diabetes, patients who were unable to understand and/or verbalize responses to the interview questions and patients who had chronic complications at advanced stages were excluded from the study.

\section{Data collection}

Adherence to therapy was measured by using the eight-item Morisky Medication Adherence questionnaire reported by Sakthong and colleagues [10]. Scores on the MMAS-8 range from zero to eight (Appendix A). A score below six indicates low adherence, while a score between six and eight indicates medium adherence and a score of eight indicates high adherence. In this study, medium and high adherence was considered as adherent and low adherence was noted as nonadherent for statistical purposes.

A separate validated questionnaire was designed to collect information on some of the probable factors affecting adherence it was validated with inputs from expert dialectologists and clinical pharmacologists before use. The questionnaire also included questions on the sociodemographic characteristics (age, sex and the educational level), duration of diabetes and barriers to patient compliance (Appendix B).

\section{Statistical analysis}

Descriptive statistics was used to find out the percentage of non-adherence and factors contributing to non-adherence. The Continuous variables are expressed as a mean (SD) and categorical variables are expressed as $\mathrm{n}(\%)$. The Chi-square test was applied to analyse categorical data. The association between level of adherence and the continuous variable was assessed by an independent sample t-test. A p value less than 0.05 was considered statistically significant .The analysis was conducted by using SPSS for Windows version 16 .

\section{Results}

The study was conducted on both genders with male participants $(48 \%)$ being slightly fewer than female participants $(52 \%)$, there was no statistically significant difference between them ( $p$ value $=0.572$ ), the majority of our participants $(54.5 \%)$ were between $40-60$ y old, while $23 \%$ of them were between $25-40$ y old and $22.5 \%$ were over 60 y of age. Sixty nine percent of patients had suffered from diabetes for over 6 years. Their educational status ranged varied between high school graduate $(51 \%)$, primary school attendance $(20 \%)$ and illiterate $(28 \%)$.

\section{Prevalence of non-adherence}

The overall prevalence of therapeutic non-adherence, that is score less than six according to the 8-item Morisky adherence scale among the participants, was $76(38 \%)$, while the majority of participants $124(62 \%)$ showed intermediate to high adherence with $\mathrm{p}$ value 0.001 .

\section{Possible barriers and insulin adherence}

Possible factor influencing patient's adherence is illustrated in Table 1.

Among the low adherent patients ( $n=76)$, the majority (44) were between 40-60 y old, while seventeen were between $25-40$ y old and fifteen patient were over $60(\mathrm{p}=0.72)$.

Forty one of the low adherent patients were at high school level, while illiteracy was seen in 22 and 13 were primary school educated $(\mathrm{p}=0.64)$. Low adherence was almost equally distributed between the male and the female (36 and 40, respectively) $(\mathrm{p}=0.9)$.

Factors that showed association with therapy non-adherence were; forgetfulness reported by thirty patients, fear of hypoglycaemia (fourteen patients) and weight gain (seven patients). 
Factors contributing to non-adherence to insulin therapy among type 1 and type 2 diabetes mellitus patients in Asser region, Saudi Arabia

Majority of the low adherent patients have been suffering from diabetes for more than 6 years $(71.7 \%)$ and they are taking more than three insulin injections/d.

A high prevalence of low adherence was seen in patients who used the pen as an injection device (44 patients). Only 7 patients who used the syringe showed low adherence and 25 patients who used other devices were also low adherent $(\mathrm{p}=0.098)$.

Patients complaint regarding interference of the therapy with physical activity, usual daily activates and meal planning was minor and was seen only in (two, five and three patients) of our participants respectively. The study also found that the following factors had minor impact on the patients adherence: only one of our participants found that the therapy was time consuming, five patients had difficulty with the injection, one patient believed that insulin caused a negative effect on overall health and one patient reported a lack of injection instructions.

Table 1. Association between possible barriers and insulin adherence in patients with types 1 and 2 diabetes.

\begin{tabular}{|c|c|c|c|}
\hline Barrier & $\begin{array}{l}\text { Low } \\
\text { adherence } \\
(n=76)\end{array}$ & $\begin{array}{l}\text { Intermediat } \\
\text { e to high } \\
\text { adherence } \\
(n=124)\end{array}$ & $\mathbf{P}$-value \\
\hline \multicolumn{4}{|l|}{ Age $(Y)$} \\
\hline $25-40$ & $17(22.4)$ & $29(23.4)$ & 0.72 \\
\hline $40-60$ & $44(57.9)$ & $65(52.4)$ & \\
\hline$>60$ & $15(19.7)$ & $30(24.2)$ & \\
\hline Educational status & & & 0.64 \\
\hline High school & $41(53.9)$ & $62(50)$ & \\
\hline Primary School & $13(17.1)$ & $28(22.6)$ & \\
\hline Illiteracy & $22(28.9)$ & $34(27.4)$ & \\
\hline Sex & & & 0.9 \\
\hline Male & $36(47.4)$ & $60(48.4)$ & \\
\hline Female & $40(52.6)$ & $64(51.6)$ & \\
\hline \multicolumn{4}{|l|}{ Diabetes characteristics } \\
\hline Duration of diabetes, Year & & & 0.357 \\
\hline $1-3$ & $12(15.8)$ & $23(18.5)$ & \\
\hline $3-6$ & $10(13.2)$ & $17(13.7)$ & \\
\hline$>6$ & $54(71.7)$ & $84(67.7)$ & \\
\hline Number of daily injections & & & 0.644 \\
\hline 1 & $15(20)$ & $23(18.9)$ & \\
\hline 2 & $36(48)$ & $52(42.6)$ & \\
\hline$\geq 3$ & $24(32)$ & $47(38.5)$ & \\
\hline Injection device & & & 0.098 \\
\hline Syringe & $7(9.2)$ & $20(16.3)$ & \\
\hline
\end{tabular}

\begin{tabular}{llll}
\hline Pen & $44(57.9)$ & $53(43.1)$ & \\
\hline Others & $25(32.9)$ & $50(40.7)$ & \\
\hline Interference with & & & \\
\hline Physical activity & $2(2.9)$ & $5(5.5)$ & 0.42 \\
\hline Usual daily activities. & $5(7.1)$ & $4(4.4)$ & 0.284 \\
\hline Meal planning & $3(4.3)$ & $3(3.3)$ & 0.441 \\
\hline Time consuming & $1(1.4)$ & $2(2.2)$ & 0.890 \\
\hline Difficulties with injection & $5(7.1)$ & $7(7.7)$ & 0.581 \\
\hline Beliefs in negative effects of insulin in & $1(1.4)$ & $0(0)$ & 0.182 \\
overall health & & & \\
\hline Embarrassment & $1(1.4)$ & $5(5.5)$ & 0.668 \\
\hline Fear of hypoglycaemia & $14(20)$ & $24(26.4)$ & 0.687 \\
\hline Weight gain & $7(10)$ & $14(15.4)$ & 0.827 \\
\hline Forgetfulness & $30(42.9)$ & $26(28.6)$ & 0.213 \\
\hline Feeling worse after insulin injection & $0(0)$ & $1(1.1)$ & 0.593 \\
\hline Lack of enough injection instructions & $1(1.4)$ & $0(0)$ & 0.182 \\
\hline Insulin shortage & $0(0)$ & $0(0)$ & 0 \\
\hline
\end{tabular}

Data are expressed as $\mathrm{n}(\%)$; $\mathrm{P}$ value significant if $<0.05$

\section{Discussion}

Adherence to prescribed anti-diabetic medications is crucial to reach metabolic control of blood glucose [11]. In spite of the importance of adherence to insulin for achieving therapeutic goals, there is lack of evidence about the rate of adherence to insulin therapy and associated risk factors [12].

Numerous studies have explored potential predictors of adherence to medicines across a variety of conditions. Frequently cited predictors include age, sex, ethnicity, income, education, and comorbidity [13].

Currently, there is no gold standard measure to evaluate medication adherence since all commonly employed methods have disadvantages. In this study, from the available methods, a self-reported 8-item Morisky's medication adherence scale was used to assess medication non-adherence. The Morisky's questionnaire was used as a method of assessing adherence as it is a simple and economic. It was also reported to be useful in clinical environments [14].

Non-adherence to treatment is a formidable problem, leading as it often does to a reduction in or lack of treatment benefits, extra visits to the doctor, unnecessary hospitalization, and decreased satisfaction with medical care and sometimes further medication prescription. This can be extremely costly, not only to the individual involved, but also to the healthcare system as a whole. Non-adherence persists regardless of the medical condition being treated and exists across socioeconomic and geographic boundaries [15].

One of the most common challenges physicians face with a patient with poorly controlled diabetes is to try to and figure 
out if the patient's hyperglycemia is due to non-adherence or is occurring despite proper medication use [13].

Even when people indicate that they have not taken all their medications as prescribed; their estimates usually substantially overestimate their actual adherence. The key validated question is 'Have you missed any pills in the past week?' and any indication of having missed one or more pills signals a problem with low adherence. Reasons for overestimation could include difficulty recalling the details of medication taking, attempting to please practitioners, to avoid confrontation, or a combination of these factors [15].

Our study showed that $38 \%$ of patients were low adherent to treatment. These results were quite low compared to other studies. For example, a study conducted in India showed that more than half of the patients were non-adherent to medication with a percentage of $54.66 \%$ [11]. This difference might be explained by patient awareness in our study and the fact that the Indian study was conducted in a hospital where majority of the patients are usually from low socioeconomic background and are less educated.

Another example is a study carried out in Nigeria where nonadherence was seen in $47.1 \%$ of patients which can also be explained by the knowledge gaps of patients with regard to indication for each of the prescribed medications, and the inaccurate description of prescribed dosage regimens among a significant proportion of patients along with their low educational level and financial problems [16].

Similarly, non-adherence was as high as 54.4\% in Sudan. These high rates were attributed mainly to forgetfulness and drug unavailability. Patients also reported some lack of knowledge about specific time of medications use [17].

However, a study conducted in 2014 showed lower rates of low adherence among patients with type 1diabetes (14.3\%), and type 2 diabetes $(28.8 \%$ ) [12]. One reason for these low rates could be the higher educational level in their study and that illiteracy was seen in only $10 \%$ of the patients compared to our $28 \%$ illiterate patients. Another reason could be the compliance with the injection device. Since most of their participants used the syringe as an injection device while most of our participants used the pen.

Various factors are associated with non-adherence to medication in DM patients, which can be categorized as patient centered, therapy-related or healthcare system related. Patientcentered factors include socio-demographic (age, gender and educational level) factors, psychological factors including motivation towards therapy taking, patient-prescriber relationship and patient knowledge. Therapy-related factors include route, type and duration of treatment, complexity of treatment especially as patients may be on multiple medicines, cost of medication and adverse effects.

Healthcare system factors include availability and accessibility of health care, and the health provider-patient interactions [18].

Although no correlation was found between low adherence and age, low adherence was mostly seen in patients between 40-60 y old (44 out of 76 low adherent patients). This finding could be due to the fact that younger patients take better care of their health to ensure a longer healthy life and the elderly seem to fear complications and mortality.

Surprisingly, most low adherent patients were high school educated (41 out of 76 low adherent patients). While primary school educated and illiterates displayed a lower frequency of low adherence. Illiteracy can interfere with understanding of the disease and medication to some extent. Studies show that the risk of non-adherence is very high when patients cannot read and understand basic written medical instructions [11]. One reason might be that lower education is accompanied with more obedience to doctor instructions.

Most low adherent patients had diabetes for over six years (54 out of 76 low adherent patients). Chronic diseases lay a burden on patients, as the long process of taking medicines becomes a heavy routine and give more chances to forgetting the medicines.

The injection device is an important factor in determining adherence to treatment, as most of the low adherent patients use the pen as an injection device while, quarter of the patients who use the syringe are low adherent and almost one third of patients who use other devices are low adherent. This result could be explained by the difficulty some patients may experience while using the pen and lack of enough instructions. Some patients may also find the syringe more familiar and convenient. Despite ease of administration and dosing accuracy of insulin pens, a study found that minority of patients $(9.1 \%)$ were using insulin pens which may be due to the great difference in medication cost compared to syringes [12].

Our results showed that among low adherent participants, the most common factors that contributed to their non-adherence were forgetfulness $40 \%$, fear of hypoglycemia $20 \%$, weight gain $10 \%$, difficulty with injection $7.1 \%$. Less common factors included interference with physical activities $4.3 \%$, patients' perception that the treatment was time consuming $1.4 \%$ and Insulin had negative effects in overall health $1.4 \%$ and embarrassment about the condition.

This study results were significantly lower than the ones obtained by another study in 2014 which indicated that $40.5 \%$ reported interference with physical activity, 20.3\% reported interference with usual daily activities, $33.8 \%$ reported interference with meal planning, $20.3 \%$ thinks it's time consuming, $21.6 \%$ have difficulty with injection, $40.5 \%$ believe in negative effects of insulin in overall health, $12.2 \%$ were 38 embarrassed, $81.1 \%$ feared hypoglycemia and $18.9 \%$ felt worse after insulin injection. In contrast, $10.8 \%$ experienced weight gain which was in accordance with our results [12]. The differences between results could be due to geographical and demographical differences. The number of participants in their study was 508 which may also play a role.

Forgetfulness seems to play a role in low adherence as 30 patients of 76 low adherent patients reported forgetfulness. Forgetfulness could be frequent problems for their age group [12]. This obstacle can be overcome by helping patients in 
organizing their medications with pillboxes and dosing alarms. Caregivers and family members can help in medication adherence in the elderly and in those who have polypharmacy.

As previously mentioned, $10 \%$ of low adherent patients experienced weight gain. To manage weight gain problem, some patients intentionally omit their insulin injections as a weight management plan. A well-integrated health system is needed to incorporate a customized program of medical nutrition therapy and physical activity in therapeutic strategies [12]. Patients should be educated about the proper use of insulin devises and how to manage hypoglycemic episodes as these two factors contributed to non-adherence in this study. Not only this but also, when necessary, patients who feels bad after the injection and those who are embarrassed about their condition should have access for psychological support or group therapy.

In a study conducted in New York City, 74\% of poorly adherent patients think that medicines are hard to take which is ten times higher than our results [13].

Almost $42 \%$ of low adherent patients did not have information about the medicines they were prescribed from doctor. Patients who did not have adequate information about their drug regimens well were probably at risk of non-adherence because they had not understood how to take their drugs causing them to miss on many occasions [11].

Unexpectedly, the majority of low adherent patients were aware what will happen if the medications are not taken regularly but they were still low adherent. Another study found that individuals with beliefs about the necessity of therapy had higher rates of adherence [13]. This difference in results lead us to attribute this low adherence to other factors such as the financial problems as more than half of low adherent patients have financial issues. It can also be attributed to their lack of information since the majority of participants did not know the common side effects of their medications.

In our study, almost $90 \%$ of patients were self-monitoring their blood glucose while in another study; only $13 \%$ of patients were self-monitoring [16].

Finally, we found that the prevalence of low adherence was low compared to other studies.

This study is not without limitations. Self-reporting methods are considered as a convenient and economical way to assess medication adherence, a review of prescription records which may lead to more accurate results and eliminate the possibility of patient's over- reporting their adherence when using direct questions for measuring compliance.

\section{Conclusion}

The current study suggests acceptable level of adherence among diabetic patients in Aseer region, Saudi Arabia. The study finding that the most common barriers to patients' adherence were forgetfulness, fear of hypoglycaemia, weight gain and difficulties with injections.

\section{Acknowledgement}

The authors would like to thank King Khalid University for their administrative support.

\section{References}

1. Sankar UV, Lipska K, Mini GK, Sarma PS, Thankappan $\mathrm{KR}$. The adherence to medications in diabetic patients in rural Kerala, India. Asia Pac J Public Health 2015; 27: 513-523.

2. International Diabetes Federation. The global burden of diabetes. Diabetes Atlas (7th Edn.). IDF Publications, Brussels, Belgium 2015.

3. Al-Nozha MM, Al-Maatouq MA, Al-Mazrou YY, AlHarthi SS, Arafah MR, Khalil MZ. Diabetes mellitus in Saudi Arabia. Saudi Med J 2004; 25: 1603-1610.

4. Joshy G, Dunn P, Fisher M, Lawrenson R. Ethnic differences in the natural progression of nephropathy among diabetes patients in New Zealand: hospital admission rate for renal complications, and incidence of end-stage renal disease and renal death. Diabetologia 2009; 52: 1474-1748.

5. World Health Organization. Global health observatory data repository. Geneva, Switzerland 2013.

6. Delamater AM. Improving patient adherence. Diab Care 2007; 30: 1107-1112.

7. Hemandez-Ronquillo L, Tellez-Zenteno JF, GardunoEspinosa J, Gonzalez-Acevez E. Factors associated with therapy noncompliance in type 2 diabetes patients. Salud Publica de Mexico 2003; 45: 191-197.

8. Sackett LD, Haynes RB, Gordon HG, Tugwell P. Textbook of clinical epidemiology. (2nd Edn.) London: Little, Brown and Company; Clinical Epidemiology. A Basic Science For Clinical Medicine 1991; 249-277.

9. Makeen H. Clinical pharmacists as medication therapy experts in diabetic clinics in Saudi Arabia-not just a perception but a need. Saudi Pharm J 2017; 25: 939-934.

10. Sakthong P, Chabunthom R, Charoenvisuthiwongs R. Psychometric properties of the Thai version of the 8-item Morisky medication adherence scale in patients with type 2 diabetes. Ann Pharmacother 2009; 43: 950-957.

11. Divya S, Pratibha N. Factors contributing to non-adherence to medication among type 2 diabetes mellitus in patients attending tertiary care hospital in South India. Asian J Pharm Clin Res 2015; 8: 274-276.

12. Farsaei S, Radfar M, Heydari Z, Abbasi F, Qorbani M. Insulin adherence in patients with diabetes: risk factors for injection omission. Prim Care Diabetes 2014; 8: 338-345.

13. Mann D, Ponieman D, Leventhal H, Halm E. Predictors of adherence to diabetes medications: the role of disease and medication beliefs. J Behav Med 2009; 32: 278-284.

14. Kassahun A, Gashe F, Mulisa E, Rike WA. Nonadherence and factors affecting adherence of diabetic patients to antidiabetic medication in Assela General Hospital, Oromia Region, Ethiopia. J Pharm Bioallied Sci 2016; 8: 124-129. 
15. Clark M. Adherence to treatment in patients with type 2 diabetes. J Diab Nurs 2004; 8: 386-391.

16. Adisa R, Fakeye T. Treatment non-adherence among patients with poorly controlled type 2 diabetes in ambulatory care settings in Southwestern Nigeria. African Health Sciences 2014; 14: 1-10.

17. EI-Hadiyah T, Madani A, Abdelrahim H, Yousif A. Factors affecting medication non-adherence in type 2 Sudanese diabetic patients. Pharmacol Pharm 2016; 7: 141-146.

18. Rwegerera G, Moshomo T, Gaenamong M, Oyewo T, Gollakota S, Mhimbira F, Fadare J, Godman B, Meyer J,
River Y. Antidiabetic medication adherence and associated factors among patients in Botswana; implications for the future. Alexandr J Med 2016; 1-15.

\section{*Correspondence to}

Dalia Almaghaslah

Department of Clinical Pharmacy College of Pharmacy

King Khalid University

Saudi Arabia 